\title{
BMJ
}

\section{Adiposity and weight change in mid-life in relation to healthy survival after age 70 in women: prospective cohort study}

\author{
Qi Sun, research associate, ${ }^{1}$ Mary K Townsend, research fellow, ${ }^{2}$ Olivia I Okereke, associate epidemiologist \\ and associate psychiatrist, ${ }^{3}$ Oscar H Franco, assistant clinical professor in public health, ${ }^{4}$ Frank B Hu, professor \\ of nutrition and epidemiology, ${ }^{1,2,3}$ Francine Grodstein, associate professor of epidemiology ${ }^{2,3}$
}

\begin{abstract}
'Department of Nutrition, Harvard School of Public Health, 655 Huntington Avenue, Boston, MA 02115, United States

${ }^{2}$ Department of Epidemiology, Harvard School of Public Health, Boston
\end{abstract}

${ }^{3}$ Channing Laboratory, Department of Medicine, Brigham and Women's Hospital and Harvard Medical School, 181 Longwood Avenue, Boston

${ }^{4} B-160$, Health Sciences Research Institute, University of Warwick, Coventry CV4 7AL

Correspondence to: Q Sun qisun@hsph.harvard.edu

Cite this as: $B M J$ 2009;339:b3796 doi:10.1136/bmj.b3796

\section{ABSTRACT}

Objective To examine the hypothesis that mid-life adiposity is associated with a reduced probability of maintaining an optimal health status among those who survive to older ages.

Design Prospective cohort study.

Setting The Nurses' Health Study, United States.

Participants 17065 women who survived until at least the age of 70 , provided information on occurrence of chronic disease, cognitive function, physical function, and mental health at older ages, and were free from major chronic diseases at mid-life (mean age was 50 at baseline in 1976).

Main outcome measures Healthy survival to age 70 and over was defined as having no history of 11 major chronic diseases and having no substantial cognitive, physical, or mental limitations.

Results Of the women who survived until at least age 70, 1686 (9.9\%) met our criteria for healthy survival. Increased body mass index (BMI) at baseline was significantly associated with linearly reduced odds of healthy survival compared with usual survival, after adjustment for various lifestyle and dietary variables ( $P<0.001$ for trend). Compared with lean women (BMI 18.5-22.9), obese women (BMI $\geq 30$ ) had $79 \%$ lower odds of healthy survival (odds ratio $0.21,95 \%$ confidence interval 0.15 to 0.29 ). In addition, the more weight gained from age 18 until mid-life, the less likely was healthy survival after the age of 70 . The lowest odds of healthy survival were among women who were overweight (BMI $\geq 25)$ at age 18 and gained $\geq 10 \mathrm{~kg}$ weight $(0.18,0.09$ to 0.36 ), relative to women who were lean (BMI 18.5-22.9) and maintained a stable weight.

Conclusions These data provide evidence that adiposity in mid-life is strongly related to a reduced probability of healthy survival among women who live to older ages, and emphasise the importance of maintaining a healthy weight from early adulthood.

\section{INTRODUCTION}

There are two current trends in industrialised countries that will have a considerable impact on public health. Firstly, the oldest age groups are growing at a rapid pace - for example, from 1900 to 2000, the population in the United States aged 75 and older increased by $26.4 \%$ to reach 16.6 million. ${ }^{1}$ In addition, there has been a steady increase in overweight and obesity; in 2003-4, 66.3\% of American adults were overweight or obese compared with $14.5 \%$ in $1976 .{ }^{23}$ Despite the evidence that overweight and obesity could significantly increase the risk of premature death, ${ }^{4-7}$ data are sparse regarding how adiposity affects overall health and wellbeing among those who survive to older ages. $^{89}$

The World Health Organization defines health as a state of complete wellbeing and not merely the absence of disease or infirmity. Similarly, Rowe and Kahn raised the concept of successful ageing to include not only disease status but also cognitive, physical, and other functions. ${ }^{10}$ Although studies have begun to address predictors of successful ageing or healthy survival, ${ }^{911}$ few have used the full spectrum of contributors from occurrence of chronic disease, cognitive, and physical functioning to mental wellness. ${ }^{8912}$ In addition, only one comprehensive study, the Honolulu Heart Program/Honolulu Asia Aging Study, has investigated adiposity at mid-life and healthy survival. They found that being overweight in mid-life was associated with a significantly reduced probability of healthy survival in men at age 85 or older. ${ }^{9}$ Importantly, although women live longer than men and tend to have a higher prevalence of overweight and obesity, ${ }^{13}$ data for women are lacking.

We investigated early adulthood and mid-life adiposity and weight change from early adulthood to midlife in relation to healthy survival at age 70 and older in the Nurses' Health Study.

\section{METHODS}

The Nurses' Health Study data collection

The Nurses' Health Study started in 1976, when 121700 female registered nurses aged 30-55 living in one of 11 US states responded to a questionnaire about history of disease and demographic and lifestyle characteristics. Since baseline, follow-up questionnaires have been administered every two years to update the information on incidence of disease and lifestyle and clinical risk factors. Starting in 1980, validated 
food frequency questionnaires have been administered every two to four years to collect and update dietary intakes of foods and nutrients. Up to 2000 (the year in which health status was determined for the analyses presented here), the follow-up rate of the entire cohort was over $95 \%$.

Self reports of major chronic diseases (such as cancer, diabetes, coronary heart disease, stroke, Parkinson's disease, and multiple sclerosis) were confirmed through various methods, including review of medical records and pathology reports, telephone interview, and supplementary questionnaires to participants. The high validity and reliability of reported incidence of chronic diseases among these nurses have been previously shown. ${ }^{13-16}$ Deaths were identified by reports from next of kin, postal authorities, or by a search of the national death index. At least $98 \%$ of deaths among the participants of the Nurses' Health Study have been identified. ${ }^{17}$

The SF-36 health status survey was included on the 1992, 1996, and 2000 questionnaires. This 36 item questionnaire measures eight health concepts, including limitations of physical activities, usual role activities, and social activities, as well as mental health, bodily pain, vitality, and the perceptions of general health. ${ }^{18}$ Its validity and reproducibility have been extensively examined, and it is commonly used to measure quality of life in different populations. ${ }^{18}$

Finally, to assess cognitive function, beginning in 1995 , we identified all nurses who had reached age 70 or older. After exclusion of nurses with a previous diagnosis of stroke, 19415 (93\%) underwent the telephone interview for cognitive status, which is modelled on the mini-mental state examination. ${ }^{19} \mathrm{~A}$ strong correlation (correlation coefficient 0.94) was documented between the scores of these two methods. ${ }^{19}$ Trained nurses who were blinded to the study hypothesis and exposure status of the participants carried out the telephone interviews. The high reliability of the interviewers and the validity of telephone assessments compared with inperson examinations have been previously shown. ${ }^{20}$ Our current analysis was conducted within this subcohort of the oldest participants of the Nurses' Health Study who were administered a cognitive function assessment.

\section{Anthropometric measures of adiposity}

Weight and height were collected on the baseline questionnaire, and weight was further requested every two years thereafter. Self reported weight was highly correlated (correlation coefficient 0.96) with measured weight in a previous validation study in 184 participants. ${ }^{21}$ We calculated the body mass index (BMI) as weight in kilograms divided by the square of height in metres $\left(\mathrm{kg} / \mathrm{m}^{2}\right)$ to measure overall obesity. In 1980, participants were asked about their weight at age 18 (on average 36 years from age 18 to year 1980 for the study participants). The correlation coefficient between recalled weight at age 18 and measured weight in physical examination records at age 18 was 0.87 among 188 participants. ${ }^{22}$ Data on BMI at age 18 were available for $89 \%$. We used waist circumference (umbilicus), hip circumference (the largest circumference), and waist to hip ratio as measured in 1986 to assess central obesity.

We chose to define adiposity measures at mid-life (that is, study baseline), both because we were interested in the relation of earlier life adiposity to health in later life and also because we were concerned about the possibility of reverse causation-that is, BMI or weight change being a consequence rather than the cause of health problems. Specifically, most of the components of our outcome can have long latency periods, and women beginning to develop these health problems might lose or gain weight. We addressed this potential bias by imposing a long followup period between baseline and outcome ascertainment and by excluding women who had had a diagnosis of the chronic diseases in our study outcome at baseline.

\section{Assessment of end points}

Although there is no consensus on the definition of successful ageing or healthy survival, the working definitions in most previous studies ${ }^{891112}$ were based on the concept raised by Rowe and Kahn, which incorporates not only chronic diseases but also physical, cognitive, and other functions. ${ }^{23}$ We used this same concept to derive our comprehensive working definition of healthy survival. Specifically, for our primary definition, healthy survivors were participants who survived to age 70 or older and as of age 70 were free from 11 major chronic diseases - that is, cancer (except nonmelanoma skin cancer), diabetes, myocardial infarction, coronary artery bypass graft surgery, congestive heart failure, stroke, kidney failure, chronic obstructive pulmonary disease, Parkinson's disease, multiple sclerosis, and amyotrophic lateral sclerosis (because cognitive function was assessed near 2000 for $99.1 \%$ of the study population, we used the disease status up to 2000 for this domain); had no major impairment of cognitive function; had no major limitation of physical functions; and had good mental health. We defined nurses who survived to the age of $\geq 70$ and did not meet these four criteria as "usual survivors." In our cohort, there were 1686 (9.9\%) "healthy survivors."

The chosen chronic conditions are major age related diseases and diseases that could severely affect the quality of life among older people. We defined impairment of cognitive function as a score less than 31 points on the telephone interview for cognitive status (about $10 \%$ of our population), according to a standard definition of impairment. ${ }^{20}$ We considered impairment of physical function, based on an existing definition, ${ }^{24}$ as presence of any of the following limitations as reported by each participant: limited at least "a little" on moderate activities as assessed by the SF-36 (such as moving a table, bowling, or pushing a vacuum cleaner; climbing one flight of stairs; walking more than 1 mile (1.6 km); walking several blocks; bathing or dressing); or limited "a lot" on the SF-36 in more difficult physical performance items (such as running; lifting heavy 
objects; lifting or carrying groceries; climbing several flights of stairs; bending, kneeling, or stooping). In total, $74 \%$ of these older women fulfilled this established definition of physical limitations. Finally, for mental health, we used the SF-36 mental health scale, which combines five questions: have you been a very nervous person?, have you felt so down in the dumps nothing could cheer you up?, have you felt calm and peaceful?, have you felt downhearted and blue?, and have you been a happy person? There were six possible responses to each item, ranging from "none of the time" to "all the time." Based on the response to these questions, a score between 1 and 6 was assigned to each question, with the score 6 indicating the best mental status and score 1 indicating the worst. We then summed these scores and rescaled them to a range of $0-100 .{ }^{25}$ Good mental health was defined as a mental health score greater than 84 (the median value in our cohort).

As there is no standard definition of healthy survival, and as the criteria we used for some of our outcomes (such as physical function) might be considered somewhat arbitrary, we investigated the robustness of our definition and further considered an alternative classification of healthy survival. This was similar to our primary definition in being free of the 11 chronic diseases, but we used a different scoring system for defining physical impairment, and we categorised all the domains by median performance: cognitive status score higher than median $(\geq 34)$, physical function score higher than median $(\geq 75)$, and mental health score higher than median $(\geq 84)$. The physical function score in this alternative classification was derived from the responses to the questions in the physical function domain of the SF-36; for each question regarding physical function, a score of 1 was assigned if the response was "yes, limited a lot," 2 if it was "yes, limited a little," or 3 if it was "no, not limited at all." We then summed the score for all questions and rescaled the total score to a range of $0-100$. With this alternative definition, $1436(8.4 \%)$ women were categorised as healthy survivors. Of these participants, 882 $(61.4 \%)$ met the criteria of the primary definition of healthy survival, thus there was some, but far from complete, overlap of the two definitions.

In addition, as BMI might be associated with survival itself ${ }^{4-7}$ and our exclusion in the primary analyses of all women who did not survive to age 70 could possibly bias findings, we constructed another secondary outcome of healthy survival. In this secondary outcome, we added the 9352 women in the cohort who did not survive to age 70 to the group of "usual survivors."

\section{Population for analysis and statistical methods}

Our exclusion criteria were a history of major chronic diseases at study baseline in 1976, including cancer, diabetes, myocardial infarction, coronary artery bypass graft surgery, stroke, kidney failure, chronic obstructive pulmonary disease, Parkinson's disease, multiple sclerosis, or amyotrophic lateral sclerosis; missing BMI at baseline; or no data on cognitive function or missing data for more than two items on the mental health scale or for more than five items on the physical function scale in the SF-36. After we excluded these participants, data from 17065 women were available for analysis.

As the waist and hip circumferences were first assessed in 1986, we used 1986 as the study baseline for the central obesity analysis and applied the same exclusion criteria. The study population for this analysis was, therefore, a subset of the primary study population (9512 for waist circumference; 9450 for hip circumference; 9438 for waist to hip ratio).

For analysis of BMI, we grouped the nurses into six categories according to their baseline BMI: < 18.5, 18.5-22.9 (reference), 23.0-24.9, 25.0-26.9, 27.0-29.9, and $\geq 30$. For analysis of weight change, we calculated weight change between age 18 and 1976 and grouped the women into five categories: lost $\geq 4.0 \mathrm{~kg}$, stable weight (reference), gained 4.0-9.9 kg, gained 10.0$14.9 \mathrm{~kg}$, gained $15.0-19.9 \mathrm{~kg}$, and gained $\geq 20 \mathrm{~kg}$. The cut-off points for the highest categories of waist circumference $(\geq 88 \mathrm{~cm})$ and waist to hip ratio $(\geq 0.80)$ were based on WHO recommendations. ${ }^{26}$ The cut-off points for the lower four categories of waist circumference and waist to hip ratio were based on quartiles of these measurements among the remaining participants. The cut-off points for hip circumference were based on quintiles.

We used logistic regressions to model the associations of each risk factor variable and the odds of healthy versus usual survival. In the current analysis, an odds ratio less than 1 denotes an "undesirable" association or reduced odds of healthy survival associated with the risk factor, while an odds ratio larger than 1 denotes a "desirable" association or an increased odds of healthy survival. In the multivariable analysis, we adjusted for baseline variables, including age at base-

Table 1| Proportion of healthy survivors and usual survivors and distribution of components of successful ageing

\begin{tabular}{lc} 
Definition & No (\%) \\
Healthy survivors & $1686(9.9)$ \\
\hline Usual survivors & $15379(90.1)$ \\
\hline No of chronic diseases*: & $4449(28.9)$ \\
\hline 1 & $1290(8.4)$ \\
\hline 2 & $340(2.2)$ \\
\hline 3 & $144(0.9)$ \\
\hline 4 or more & $6320(41.1)$ \\
\hline No of limitations in cognitive, physical, or mental health domains*: \\
\hline 1 domain only & $7559(49.2)$ \\
\hline 2 domains & $989(6.4)$ \\
\hline 3 domains & $511(3.3)$ \\
\hline $\begin{array}{l}\text { Having one or more chronic diseases and no } \\
\text { limitations in cognitive, physical, or mental health } \\
\text { domains* }\end{array}$ & \\
\hline $\begin{array}{l}\text { Having limitations in cognitive, physical, or } \\
\text { mental health domains only and no chronic } \\
\text { diseases* }\end{array}$ & $9156(59.5)$ \\
\hline $\begin{array}{l}\text { Having both chronic disease(s) and limitation(s) } \\
\text { in cognitive, physical, or mental health domains* }\end{array}$ & $5712(37.1)$ \\
*Proportions are among usual survivors only. & \\
\hline
\end{tabular}


Table 2 | Baseline characteristics (in 1976) of healthy survivors and usual survivors in Nurses' Health Study*

\begin{tabular}{|c|c|c|c|}
\hline Characteristics & $\begin{array}{l}\text { Healthy survivor } \\
\qquad(\mathrm{n}=1686)\end{array}$ & $\begin{array}{l}\text { Usual survivor } \\
\qquad(\mathrm{n}=15 \text { 379) }\end{array}$ & $P$ value \\
\hline Age (years) & $50.1(2.5)$ & $50.5(2.5)$ & $<0.001$ \\
\hline BMI at age 18 & $21.0(2.4)$ & $21.3(2.9)$ & $<0.001$ \\
\hline BMI at baseline & $22.9(2.8)$ & $24.4(4.0)$ & $<0.001$ \\
\hline Weight change from age 18 to baseline $(\mathrm{kg})$ & $5.1(7.6)$ & $8.2(9.7)$ & $<0.001$ \\
\hline Waist circumference $(\mathrm{cm})$ & $76.2(8.3)$ & $80.6(10.3)$ & $<0.001$ \\
\hline Hip circumference $(\mathrm{cm})$ & $98.1(7.8)$ & $101.4(9.5)$ & $<0.001$ \\
\hline Waist:hip ratio & $0.78(0.08)$ & $0.79(0.07)$ & $<0.001$ \\
\hline Physical activity (hour/week) $\dagger$ & $4.3(2.9)$ & $3.6(2.8)$ & $<0.001$ \\
\hline Saturated fat (\% of energy) & $10.7(2.2)$ & $11.5(2.4)$ & $<0.001$ \\
\hline Polyunsaturated fat (\% of energy) & $5.5(1.1)$ & $5.7(1.2)$ & $<0.001$ \\
\hline Polyunsaturated:saturated fat ratio & $0.6(0.2)$ & $0.5(0.1)$ & $<0.001$ \\
\hline Trans fat (\% of energy) & $1.6(0.5)$ & $1.7(0.5)$ & $<0.001$ \\
\hline Alcohol intake (g/day) & $6.4(9.0)$ & $5.7(8.9)$ & 0.004 \\
\hline Cereal fibre (g/day) & $5.3(2.2)$ & $4.9(2.2)$ & $<0.001$ \\
\hline Red meat (serving/day) & $0.7(0.4)$ & $0.9(0.5)$ & $<0.001$ \\
\hline Fruits and vegetables (serving/day) & $5.8(1.9)$ & $5.5(1.9)$ & $<0.001$ \\
\hline \multicolumn{4}{|l|}{ Smoking statusł: } \\
\hline Never smoked & $908(54.1)$ & 7093 (46.4) & \multirow{5}{*}{$<0.001$} \\
\hline Past smoker & $433(25.8)$ & 3801 (24.9) & \\
\hline Currently smoke 1-14 cigarettes/day & $135(8.1)$ & $1349(8.8)$ & \\
\hline Currently smoke $15-24$ cigarettes/day & $132(7.9)$ & $1922(12.6)$ & \\
\hline Currently smoke $\geq 25$ cigarettes/day & $69(4.1)$ & $1121(7.3)$ & \\
\hline \multicolumn{4}{|l|}{ Education (\%): } \\
\hline Registered nurse & $1266(75.1)$ & $12324(80.1)$ & \multirow{4}{*}{$<0.001$} \\
\hline Bachelor's degree & $282(16.7)$ & $2184(14.2)$ & \\
\hline Master's degree & $126(7.5)$ & $822(5.3)$ & \\
\hline Doctoral degree & $12(0.7)$ & $49(0.3)$ & \\
\hline \multicolumn{4}{|l|}{ Husband's education (\%)‡: } \\
\hline Less than high school & $338(2.7)$ & $32(2.3)$ & \multirow{5}{*}{$<0.001$} \\
\hline Some high school & $796(6.4)$ & $79(5.6)$ & \\
\hline High school graduate & 5378 (43.3) & $552(39.0)$ & \\
\hline College graduate & $3423(27.6)$ & $412(29.1)$ & \\
\hline Graduate school & $2485(20.0)$ & $342(24.4)$ & \\
\hline \multicolumn{4}{|l|}{ Marital status (\%): } \\
\hline Married & $1076(63.8)$ & $9485(61.7)$ & \multirow{3}{*}{0.11} \\
\hline Widowed & $547(32.4)$ & $5391(35.1)$ & \\
\hline Separated/divorced/never married & $63(3.7)$ & $503(3.3)$ & \\
\hline \multicolumn{4}{|l|}{ Postmenopausal hormone use (\%)‡: } \\
\hline Never use & $497(32.8)$ & $4060(29.7)$ & \multirow{3}{*}{$<0.001$} \\
\hline Current use & $579(38.2)$ & $4989(36.5)$ & \\
\hline Past use & $437(28.9)$ & $4603(33.7)$ & \\
\hline \multicolumn{4}{|l|}{ Family history (\%): } \\
\hline Heart disease & $258(15.3)$ & $2734(17.8)$ & 0.01 \\
\hline Diabetes & $433(25.7)$ & $4510(29.3)$ & 0.002 \\
\hline Cancer & $269(16.0)$ & $2666(17.3)$ & 0.15 \\
\hline
\end{tabular}

*Values are mean (SD) for continuous variables or number (percentage) for categorical variables.

†Mostly moderate physical activity, such as walking.

$\ddagger$ Proportions are based on non-missing values. postmenopausal hormone use (never used, past user, or current user), smoking status (never smoked, past smoker, current smoker of 1-14 cigarettes a day, 1524 cigarettes a day, or $\geq 25$ cigarettes day), family history of heart disease (yes, no), family history of diabetes (yes, no), family history of cancer (yes, no), physical activity (hours a week), ratio of intake of polyunsaturated to saturated fat (in fifths), intakes of trans fat, alcohol, and cereal fibre (all in fifths), and intakes of fruits, vegetables, and red meat (in thirds) to control for confounding. When examining the associations for weight change, we further adjusted for BMI at age 18 . In analyses of waist and hip circumference, we adjusted for BMI in 1986 and mutually adjusted for waist and hip circumferences.

Cigarette smoking could reduce body weight and has strong effects on overall health. ${ }^{5}$ To account for the possibility of residual confounding by smoking we conducted a secondary analysis among women who had never smoked. In an additional secondary analysis, we adjusted for potential confounding factors defined at age 70 rather than at baseline, but this did not materially change our findings. Finally, we repeated the analyses using the alternative definitions of healthy survival and usual survival as described above.

In our examination of the joint associations of BMI at age 18 and weight change from age 18 to baseline, we included only those who had stable weight or gained weight since age 18 and whose BMI was no less than 18.5 to yield more stable estimates because only a small number of the nurses lost more than $4 \mathrm{~kg}$ body weight or were underweight at age 18. A secondary analysis showed that including these women did not change the results materially. We used likelihood ratio tests to evaluate the significance of interactions between BMI and weight change. These tests are based on the difference of $-2 \log$ likelihood of models with and without interaction terms and follow the $\chi^{2}$ distribution with the degree of freedom equal to the number of parameters in the interaction terms.

All $\mathrm{p}$ values were two sided. Odds ratios were calculated with $95 \%$ confidence intervals. Data were analysed with the SAS software package, version 9.1 (S AS Institute, Cary, NC).

\section{RESULTS}

Distribution of study outcome and baseline characteristics Of 17065 nurses who survived to age 70 or older, 1686 $(9.9 \%)$ met our primary criteria of "healthy survivor" (table 1) and 15379 (90.1\%) were "usual survivors." Among the usual survivors, $5712(37.1 \%)$ had both chronic diseases and limitations in cognitive, physical, or mental health; 9156 (59.5\%) had limitations in cognitive, physical, or mental health only; and $511(3.3 \%)$ had one or more chronic diseases only. The most common chronic diseases were cancer (except non-melanoma skin cancer) ( $\mathrm{n}=2549,16.6 \%)$, coronary heart disease (myocardial infarction and coronary artery bypass graft surgery) $(\mathrm{n}=1727,11.2 \%)$, and diabetes $(\mathrm{n}=1650,10.7 \%)$. With respect to individual functional line (year), education (registered nurse certificate bachelor's degree, master's degree, or doctoral degree), husband's education (less than high school, some high school, high school graduate, college graduate, or graduate school), marital status (married, widowed, separated/divorced/never married), 
Table 3| Odds ratios (95\% confidence interval) of healthy survival associated with baseline (1976) BMI (body mass index) in Nurses' Health Study

\begin{tabular}{|c|c|c|c|c|c|c|c|}
\hline & $<18.5$ & $18.5-22.9$ & $23.0-24.9$ & $25.0-26.9$ & $27.0-29.9$ & $\geq 30$ & P for trend* \\
\hline Case/No & $31 / 187$ & $918 / 6145$ & $413 / 3700$ & $188 / 2234$ & $98 / 1749$ & $38 / 1364$ & - \\
\hline Age adjusted & 1.13 (0.76 to 1.64$)$ & 1.0 & $0.76(0.67$ to 0.86$)$ & 0.58 (0.49 to 0.68$)$ & $0.38(0.31$ to 0.47$)$ & $0.19(0.14$ to 0.26$)$ & $<0.001$ \\
\hline Multivariable† & 1.29 (0.87 to 1.92$)$ & 1.0 & 0.76 (0.67 to 0.87$)$ & 0.58 (0.49 to 0.69$)$ & $0.40(0.32$ to 0.50$)$ & 0.21 (0.15 to 0.29$)$ & $<0.001$ \\
\hline
\end{tabular}

*Estimates of $\mathrm{P}$ value for linear trend based on linear scores derived from medians of each BMI category.

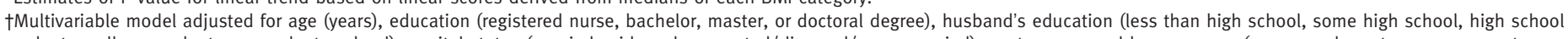

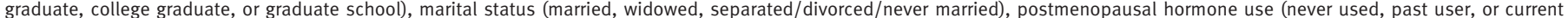

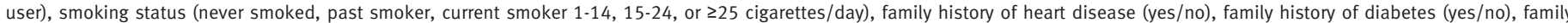

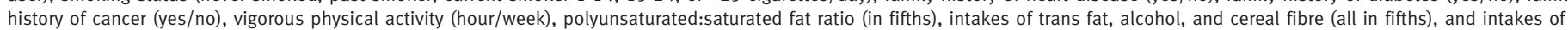
fruits and vegetables and red meat (in thirds), all defined at baseline.

limitations based on our primary definitions, 1832 (10.7\%) had cognitive impairments, 12641 (74.1\%) had physical limitations, and $9932(58.2 \%)$ had less optimal mental health status.

Table 2 shows the baseline characteristics of healthy survivors and usual survivors in our primary analyses. As expected, in comparison with usual survivors, healthy survivors were healthier at baseline, with a lower prevalence of cigarette smoking and somewhat better diet. In addition, we found that healthy survivors had received more education than usual survivors. With respect to adiposity, healthy survivors were less likely to have overall or central obesity at baseline and tended to gain less weight since age 18 than usual survivors.

Overall obesity, weight gain, and healthy survival

Tables 3 and 4 show the odds ratios of healthy survival according to $\mathrm{BMI}$ at baseline and weight change from age 18 to baseline. After adjustment for multiple potential confounding factors, we found linearly reduced odds of healthy survival associated with increasing BMI at baseline. Every one unit increase of BMI was associated with a $12 \%$ reduction of the odds of healthy survival (95\% confidence interval $10 \%$ to $14 \%)$. Similarly, in comparison with stable weight, weight gain since age 18 was significantly associated with reduced odds of healthy survival. Although relatively few women lost weight, there was a small, non-significant increase in the odds of healthy survival in women who had lost weight between age 18 and baseline compared with women with stable weight (odds ratio 1.18, 0.94 to 1.46). For every $1 \mathrm{~kg}$ increase of weight gain since age 18 , the odds of healthy survival decreased by $5 \%(4 \%$ to $6 \%$. The associations we found for baseline BMI and for weight change persisted among never smokers, indicating that residual confounding by smoking could not explain our findings. For example, among women who had never smoked, the odds ratio for those with $\mathrm{BMI} \geq 30$ at baseline was $0.27(0.18$ to $0.39 ; \mathrm{P}<0.001$ for trend) compared with lean women (BMI 18.5-22.9). With respect to weight change, among those who had never smoked, women who gained $\geq 20 \mathrm{~kg}$ had an odds ratio of 0.16 (0.11 to 0.25 ; $\mathrm{P}<0.001$ for trend) compared with women who maintained a stable weight. Higher BMI at age 18 was also significantly associated with reduced odds of healthy survival. After multivariable adjustment of covariates, relative to women with $\mathrm{BMI}$ of $18.5-22.9$ at age 18 , the odds ratios for healthy survival were 0.85 (0.71 to 1.01$)$ for those with BMI of 23.0-24.9 and 0.67 (0.54 to 0.84) for those with $\mathrm{BMI} \geq 25$, respectively $(\mathrm{P}=0.001$ for trend).

The worst odds ratio for healthy survival was found among nurses who were overweight at age 18 and gained $\geq 10 \mathrm{~kg}$ between age 18 and baseline (figure). Compared with women with a BMI of 18.5-22.9, who also had stable weight over time, women in the aforementioned group had an odds ratio of 0.18 (0.09 to 0.36). Within each BMI category at age 18 , those who gained more weight had lower odds of healthy survival. Even among women who were lean (BMI 18.522.9) at age 18, relative to those who kept a stable weight women who gained $4.0-9.9 \mathrm{~kg}$ had an odds ratio of 0.67 ( 0.58 to 0.78 ) and women who gained more than $10 \mathrm{~kg}$ had an odds ratio of 0.41 ( 0.35 to 0.50). We did not, however, find any significant interaction between $\mathrm{BMI}$ at age 18 and subsequent weight gain $(\mathrm{P}=0.35$ for interaction $)$.

\section{Central obesity and healthy survival}

After adjustment for covariates and mutual adjustment for each other, increased waist circumference (table 5) and hip circumference (table 6) were each associated with reduced odds of healthy survival. Increased waist to hip ratio was also associated with lower odds of healthy survival after adjustment for BMI and other covariates (table 7).

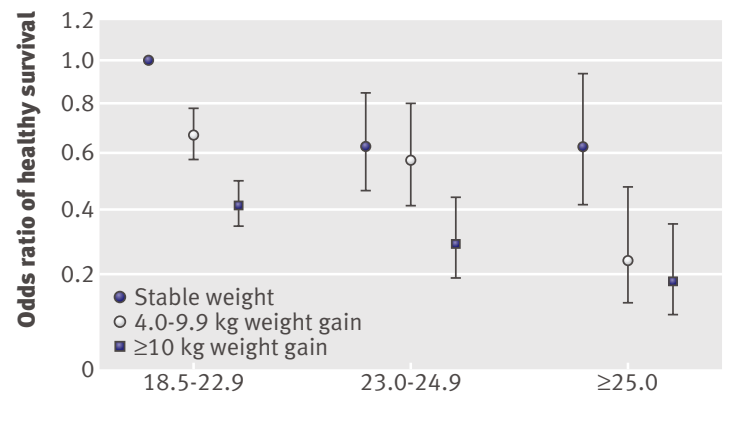

BMI at age 18

Joint effect of BMI at age 18 and weight change on healthy survival in the Nurses' Health study. Adjusted odds with $95 \%$ confidence intervals (see table 3 ) 
Table 4 | Odds ratios (95\% confidence interval) of healthy survival associated with weight change since age 18 in Nurses' Health Study

\begin{tabular}{|c|c|c|c|c|c|c|c|}
\hline & Loss $\geq 4 \mathrm{~kg}$ & Stable & Gain 4.0-9.9 kg & Gain $10.0-14.9 \mathrm{~kg}$ & Gain $15.0-19.9 \mathrm{~kg}$ & Gain $\geq 20 \mathrm{~kg}$ & P for trend* \\
\hline Case/No & $140 / 1022$ & $555 / 3417$ & $501 / 4360$ & $198 / 2194$ & $90 / 1224$ & $43 / 1483$ & - \\
\hline Age adjusted & 0.84 (0.69 to 1.03$)$ & 1.0 & 0.71 (0.63 to 0.81$)$ & $0.56(0.48$ to 0.67$)$ & 0.47 (0.37 to 0.59$)$ & $0.18(0.13$ to 0.25$)$ & $<0.001$ \\
\hline Multivariable†‡ & 1.18 (0.94 to 1.46$)$ & 1.0 & $0.66(0.58$ to 0.76$)$ & 0.53 (0.44 to 0.63$)$ & $0.45(0.35$ to 0.57$)$ & $0.19(0.13$ to 0.26$)$ & $<0.001$ \\
\hline
\end{tabular}

*Estimates of $\mathrm{P}$ value for linear trend are based on linear scores derived from the medians of each weight change category.

†Multivariable model adjusted as in table 3.

$\ddagger$ Additionally adjusted for BMI at age 18 ( $(18.5,18.5-24.9$, or $\geq 25)$.

\section{Secondary analyses}

We further examined the associations between adiposity and the alternative definitions of healthy survival. When we redefined healthy survival, with medians as the cut-off points for cognitive, physical, and mental health, we found similar associations as in our primary analyses (data not shown in table), indicating that our definition of healthy survival is robust to variations in the cut-off points and scales used for health domains. For example, the odds ratios associated with the alternative definition were 0.81 (0.71 to 0.92 ) for BMI of $23.0-24.9 ; 0.67$ (0.57 to 0.80 ) for BMI of 25.0-26.9; 0.47 (0.37 to 0.59) for BMI of 27.0-29.9; and 0.22 $(0.15$ to 0.32$)$ for $\mathrm{BMI} \geq 30$ at baseline $(\mathrm{P}<0.001$ for trend).

In additional secondary analyses, in which we included the 9352 women who did not survive to age 70 in the comparison group of "usual survivors," associations did not materially change. For example, compared with the reference group (BMI 18.5-22.9), the odds ratio of healthy survival in these analyses was 0.75 (0.66 to 0.85) for women with BMI 23.0-24.9; 0.55 (0.47 to 0.65) for women with BMI 25.0-26.9; 0.35 (0.28 to 0.44) for women with BMI 27.0-29.9; and 0.16 (0.12 to 0.23 ) for women with $\mathrm{BMI} \geq 30$. With respect to weight change, the odds ratios were 0.67 (0.59 to 0.77 ), 0.50 (0.42 to 0.60$), 0.40$ (0.31 to $0.50)$, and 0.15 (0.11 to 0.21$)$ for women who gained $4.0-9.9 \mathrm{~kg}, 10.0-14.9 \mathrm{~kg}, 15.0-19.9 \mathrm{~kg}$, and $\geq 20 \mathrm{~kg}$, respectively. Thus, there did not seem to be meaningful bias induced by excluding women who did not survive to age 70 in our primary analyses.

\section{DISCUSSION}

In this established cohort of registered nurses, mid-life adiposity strongly predicted impaired overall health, as assessed by incidence of chronic diseases and a full spectrum of physical, cognitive, and mental health outcomes among participants who survived to age 70 or older. In addition, both overweight in early adulthood and weight gain from early adulthood to mid-life were independently and significantly associated with reduced odds of healthy survival versus usual survival. These data, in combination with data from studies of adiposity and survival, provide important evidence that maintaining a low BMI through mid-life not only lowers mortality but also enhances overall health in those who survive to older ages.

\section{Strengths and limitations}

We constructed a comprehensive definition of healthy survival, including chronic diseases, physical limitations, cognitive impairment, and mental wellbeing. By excluding women who had chronic diseases at baseline and by using baseline BMI only, we minimised the possibility of reverse causation - that body weight might be a consequence, rather than a cause, of underlying health conditions. In addition, in the current analysis, we controlled for a wide array of potential confounders, such as socioeconomic status (as represented by the educational attainment of the nurses and their husbands) and demographic, lifestyle, and dietary risk factors. We further restricted some analyses to women who never smoked to diminish any residual confounding by smoking status and found similar results. Other strengths include the high follow-up rate, large sample size, valid data on chronic diseases, and validated methods to measure physical and mental limitations and cognitive function. In addition, the rich dataset allowed us to perform detailed analyses on adiposity in relation to healthy survival.

The study population was primarily white, working nurses $(94.2 \%$ were white with European ancestry) with relatively better health status and behaviours than the general population. Although the homogeneity of healthcare access within this population helps to reduce some confounding and enhances the internal validity, the results might not be generalisable to all populations, especially populations other than white professionals. In addition, although self reported current body weight was highly accurate, ${ }^{21}$ self reported weight at age 18 might have introduced some

Table $5 \mid$ Odds ratios ( $95 \%$ confidence interval) of healthy survival associated with waist circumference assessed in 1986 in Nurses' Health Study*

\begin{tabular}{|c|c|c|c|c|c|c|}
\hline & $<71 \mathrm{~cm}$ & $71-75 \mathrm{~cm}$ & $76-80 \mathrm{~cm}$ & $81-87 \mathrm{~cm}$ & $\geq 88 \mathrm{~cm}$ & P for trend $\dagger$ \\
\hline Case/No & $232 / 1081$ & $304 / 1642$ & $238 / 1683$ & $211 / 2208$ & $104 / 1809$ & - \\
\hline Age adjusted & 1.0 & $0.87(0.72$ to 1.05$)$ & 0.66 (0.54 to 0.81$)$ & 0.45 (0.37 to 0.55$)$ & $0.27(0.21$ to 0.35$)$ & $<0.001$ \\
\hline Multivariable $\ddagger$ & 1.0 & 0.87 (0.71 to 1.07$)$ & 0.74 (0.58 to 0.93$)$ & 0.59 (0.45 to 0.77$)$ & $0.45(0.32$ to 0.63$)$ & $<0.001$ \\
\hline
\end{tabular}

*Baseline was 1986.

†Estimates of $\mathrm{P}$ value for linear trend based on linear scores derived from medians of each waist circumference category.

$\ddagger$ Multivariable model adjusted as in table 3 plus BMI and hip circumference at baseline. 
Table 6 | Odds ratios (95\% confidence interval) of healthy survival associated with hip circumferences assessed in 1986 in Nurses' Health Study*

\begin{tabular}{|c|c|c|c|c|c|c|}
\hline & $<91 \mathrm{~cm}$ & $91-96 \mathrm{~cm}$ & $97-101 \mathrm{~cm}$ & $102-106 \mathrm{~cm}$ & $\geq 106 \mathrm{~cm}$ & $\mathrm{P}$ for trend $\dagger$ \\
\hline Case/No & $237 / 1318$ & $336 / 1868$ & $273 / 2000$ & $138 / 1495$ & $100 / 1658$ & - \\
\hline Age adjusted & 1.0 & $1.01(0.84$ to 1.20$)$ & 0.77 (0.64 to 0.93$)$ & $0.52(0.41$ to 0.65$)$ & $0.33(0.26$ to 0.42$)$ & $<0.001$ \\
\hline Multivariable $\ddagger$ & 1.0 & 1.08 (0.89 to 1.32$)$ & $0.97(0.77$ to 1.21$)$ & 0.77 (0.58 to 1.02$)$ & 0.67 (0.47 to 0.94$)$ & 0.01 \\
\hline
\end{tabular}

*Baseline was 1986

†Estimates of $P$ value for linear trend based on linear scores derived from medians of each hip circumference category.

$\ddagger$ Multivariable model adjusted as in table 3 plus BMI and waist circumference at baseline.

misclassification. In a validation study in the Nurses' Health Study II, however, the difference between measured and self reported body weight at age 18 was, on average, only $1.4 \mathrm{~kg} .{ }^{22}$ As the recall of remote body weight was independent of the study outcome, such misclassification was probably random and the true associations might be underestimated here. At the study end point, most women were under the age of 75 (median age 74). Whether these associations would persist among considerably older women is unknown. In the Honolulu Heart Program/Honolulu Asia Aging Study cohort, however, overweight at midlife was also associated with a reduced probability of healthy survival among men age 85 and older. ${ }^{9}$

An additional limitation was the subjectivity in our definition of healthy survivor. There is currently no standard definition and so we were forced to make "subjective" decisions regarding our definition. For example, we chose not to include osteoporosis, hip fracture, or hip replacement in the definition of healthy survival. This was because our physical function domain reflects the severity and meaningful consequences of these conditions and thus we thought it could be redundant to include them. In addition, the cut-off points we used to define physical limitations, cognitive impairments, and mental limitations could be considered somewhat arbitrary. When we examined the associations using alternative cut-off points, however, we found similar results, indicating that our findings were not completely dependent on the cut-off points we chose. Thus, overall, our primary definition of healthy survival seemed to be meaningful and rational. Though nurses with chronic diseases or functional limitations might have been lost to follow-up; the overall high follow-up rate for the study minimises any meaningful impact of such bias on our results. Lastly, as our study is observational in nature, part of the observed associations might be explained by confounding, although we believe we have reduced confounding as much as possible, as discussed above.

\section{Results in relation to other studies}

The findings in our study were consistent with the few previous studies that examined BMI and healthy survival in men of Japanese ancestry. ${ }^{89}$ In the current analysis in women, our data provided new evidence suggesting the absence of any threshold effects of excessive body weight on healthy survival; the probability of healthy survival started falling linearly even when mid-life BMI was still within a "normal" range (18.5-25.0). In addition, our data were also in line with accumulating evidence that suggests central or visceral obesity, as measured by waist circumference or waist to hip ratio, could predict multiple adverse health outcomes, including cognitive decline and physical limitations, even beyond the effects of overall obesity. ${ }^{427-31}$ Interestingly, in our analysis, regardless of the BMI at early adulthood, weight gain during adulthood was clearly a risk factor predicting adverse health status at older ages. Even a moderate weight gain of $4-10 \mathrm{~kg}$ was significantly associated with reduced odds of healthy survival. These observations were consistent with our previous findings on individual outcomes, that weight gain since early adulthood was associated with increased risks of developing type 2 diabetes, coronary heart disease, or premature death. ${ }^{5132}$ Together with the observation that even $\mathrm{BMI}$ at age 18 was associated with moderately, albeit significantly, reduced odds of healthy survival at much older ages, these data emphasised the significance of maintaining a healthy weight throughout adulthood to enjoy a long and healthy life.

\section{Conclusions}

In summary, this study provides new evidence that greater adiposity at mid-life is a strong risk factor

Table $7 \mid$ Odds ratios (95\% confidence interval) of healthy survival associated with waist to hip ratio assessed in 1986 in Nurses' Health Study*

\begin{tabular}{lcccccc} 
& $<0.73$ & $0.73-0.75$ & $0.76-0.77$ & $0.78-0.79$ & P & P for trend $\dagger$ \\
Case/No & $242 / 1292$ & $237 / 1352$ & $143 / 1103$ & $153 / 1157$ & $308 / 3451$ & - \\
\hline Age adjusted & 1.0 & $0.94(0.77$ to 1.14$)$ & $0.70(0.56$ to 0.87$)$ & $0.71(0.57$ to 0.88$)$ & $0.49(0.41$ to 0.58$)$ & $<0.001$ \\
\hline Multivariable & 1.0 & $1.00(0.82$ to 1.22$)$ & $0.83(0.66$ to 1.04$)$ & $0.84(0.67$ to 1.05$)$ & $0.68(0.56$ to 0.83$)$ & $<0.001$
\end{tabular}

*Baseline was 1986

†Estimates of $\mathrm{P}$ value for linear trend based on linear scores derived from medians of each waist:hip ratio category.

¥Multivariable model adjusted as in table 3 plus BMI at baseline. 


\section{WHAT IS ALREADY KNOWN ON THIS TOPIC}

Adiposity is associated with increased risk of many chronic diseases and premature death

Few studies have been conducted to elucidate whether mid-life adiposity is also associated with a reduced probability of maintaining an overall optimal health status among those who escaped premature death, especially in women

\section{WHAT THIS STUDY ADDS}

Increased adiposity, as well as weight gain since early adulthood, in middle aged women was associated with a linearly decreased probability of healthy survival at age 70 and over, as defined by an absence of major chronic diseases and physical, cognitive, and mental limitations in older ages

It is important to maintain a healthy weight from early adulthood to enjoy a healthy life in older ages
8 Reed DM, Foley DJ, White LR, Heimovitz H, Burchfiel CM, Masaki K. Predictors of healthy aging in men with high life expectancies. Am J Public Health 1998;88:1463-8.

9 Willcox BJ, He Q, Chen R, Yano K, Masaki KH, Grove JS, et al. Midlife risk factors and healthy survival in men. JAMA 2006;296:2343-50.

10 Rowe JW, Kahn RL. Successful aging. Gerontologist 1997;37:433-40.

11 Depp CA, Glatt SJ, Jeste DV. Recent advances in research on successful or healthy aging. Curr Psychiatry Rep 2007;9:7-13.

12 Newman AB, Arnold AM, Naydeck BL, Fried LP, Burke GL, Enright P, et al. "Successful aging": effect of subclinical cardiovascular disease. Arch Intern Med 2003;163:2315-22.

13 Colditz GA, Willett WC, Rotnitzky A, Manson JE. Weight gain as a risk factor for clinical diabetes mellitus in women. Ann Intern Med 1995;122:481-6.

14 Barr RG, Herbstman J, Speizer FE, Camargo CA Jr. Validation of selfreported chronic obstructive pulmonary disease in a cohort study of nurses. Am J Epidemiol 2002;155:965-71.

15 Alonso A, Hernan MA, Ascherio A. Allergy, family history of autoimmune diseases, and the risk of multiple sclerosis. Acta Neurol Scand 2008;117:15-20.

16 Sun Q, Ma J, Campos H, Hankinson SE, Manson JE, Stampfer MJ, et al. A prospective study of trans fatty acids in erythrocytes and risk of coronary heart disease. Circulation 2007;115:1858-65.

17 Stampfer MJ, Willett WC, Speizer FE, Dysert DC, Lipnick R, Rosner B, et al. Test of the National Death Index. Am J Epidemiol 1984;119:837-9.

18 Ware JE, Snow KK, Kosinski M, Gandek B. SF-36 health survey: manual and interpretation guide. Boston: The Health Institute, New England Medical Center, 1993.

19 Brandt J, Spencer M, Folstein M. The telephone interview for cognitive status. Neuropsychiatry Neuropsychol Behav Neurol 1988;1:111-7.

20 Stampfer MJ, Kang JH, Chen J, Cherry R, Grodstein F. Effects of moderate alcohol consumption on cognitive function in women. $N$ Engl J Med 2005;352:245-53.

21 Willett W, Stampfer MJ, Bain C, Lipnick R, Speizer FE, Rosner B, et al. Cigarette smoking, relative weight, and menopause. Am J Epidemiol 1983;117:651-8.

22 Troy LM, Hunter DJ, Manson JE, Colditz GA, Stampfer MJ, Willett WC. The validity of recalled weight among younger women. Int J Obes Relat Metab Disord 1995;19:570-2.

23 Rowe JW, Kahn RL. Human aging: usual and successful. Science 1987;237:143-9.

24 Strawbridge WJ, Cohen RD, Shema SJ, Kaplan GA. Successful aging: predictors and associated activities. Am J Epidemiol 1996;144:135-41.

25 Michael YL, Berkman LF, Colditz GA, Kawachi I. Living arrangements, social integration, and change in functional health status. Am J Epidemiol 2001;153:123-31.

26 Obesity: preventing and managing the global epidemic. Report of a WHO consultation. World Health Organ Tech Rep Ser 2000;894:i-xii,1-253.

27 Chan JM, Rimm EB, Colditz GA, Stampfer MJ, Willett WC. Obesity, fat distribution, and weight gain as risk factors for clinical diabetes in men. Diabetes Care 1994;17:961-9.

28 Folsom AR, Kushi LH, Anderson KE, Mink PJ, Olson JE, Hong CP, et al. Associations of general and abdominal obesity with multiple health outcomes in older women: the lowa Women's Health Study. Arch Intern Med 2000;160:2117-28.

29 Janssen I, Katzmarzyk PT, Ross R. Body mass index, waist circumference, and health risk: evidence in support of current National Institutes of Health guidelines. Arch Intern Med 2002;162:2074-9.

30 Houston DK, Stevens J, Cai J. Abdominal fat distribution and functional limitations and disability in a biracial cohort: the Atherosclerosis Risk in Communities Study. Int J Obes (Lond) 2005;29:1457-63.

31 Whitmer RA, Gustafson DR, Barrett-Connor E, Haan MN, Gunderson EP, Yaffe K. Central obesity and increased risk of dementia more than three decades later. Neurology 2008.

32 Li TY, Rana JS, Manson JE, Willett WC, Stampfer MJ, Colditz GA, et al. Obesity as compared with physical activity in predicting risk of coronary heart disease in women. Circulation 2006;113:499-506.

Accepted: 22 June 2009 